\title{
The Helpful Environment: distributed agents and services which cooperate
}

\author{
Austin Tate \\ Artificial Intelligence Applications Institute, University of Edinburgh, \\ Appleton Tower, Crichton Street, Edinburgh EH8 9LE, UK \\ a.tate@ed.ac.uk
}

\begin{abstract}
Imagine a future environment where networks of agents - people, robots and software agents - interact with sophisticated sensor grids and environmental actuators to provide advice, protection and aid. The systems will be integral to clothing, communications devices, vehicles, transportation systems, buildings, and pervasive in the environment. Vehicles and buildings could assist both their occupants and those around them. Systems would adapt and respond to emergencies whether communication were possible or not. Where feasible, local help would be used, with appropriate calls on shared services facilitated whenever this is both possible and necessary. Through this framework requests for assistance could be validated and brokered to available and appropriate services in a highly distributed fashion. Services would be provided to individuals or communities through this network to add value and give all sorts of assistance beyond emergency response aspects. In emergency situations, the local infrastructure would be augmented by the facilities of the responder teams at any level from local police, ambulance and fire response, all the way up to international response. An emergency zone's own infrastructure could be augmented when necessary by laying down temporary low cost sensor grids and placing specialized devices and robotic responders into the disaster area. These would form the basis for a distributed, adaptable, and resilient "helpful environment” for every individual and organisation at personal, family, business, regional, national and international levels.
\end{abstract}

Keywords: Intelligent agents, distributed systems, collaborative systems, cooperative systems, sensor grids, emergency response.

\section{Introduction}

Imagine a future environment where a network of agents - people, robots and software agents - interact with sophisticated sensor grids and environmental actuators to provide advice, protection and aid. The systems will be integral to clothing, communications devices, vehicles, transportation systems, buildings, and pervasive in the environment. These would form the basis for a distributed, adaptable, and resilient "helpful environment" for every individual and organisation at personal, family, business, regional, national and international levels. In natural disaster-prone areas government legislation, building codes and insurance requirements would ensure that appropriate 
sensor/actuator systems were included in future communication devices, vehicles and buildings to assist both their users and those around them. Systems would adapt and respond to emergencies whether communication were possible or not. Where feasible, local help would be used, with appropriate calls on shared services facilitated whenever this is both possible and necessary. Through this framework requests for assistance could be validated and brokered to available and appropriate services in a highly distributed market fashion. Services would be provided to individuals or communities through this network to add value and give all sorts of assistance beyond the emergency response aspects. In emergency situations, the local infrastructure would be augmented by the facilities of the responder teams at any level from local police, ambulance and fire response, all the way up to international response. An emergency zone's own infrastructure could be augmented on demand by laying down temporary low cost sensor grids and placing specialized devices and robotic responders into the disaster area.

\section{Emergency Response Challenges}

Local or regional governments are often responsible for the event handling, planning, coordination and status reporting involved in responding to an emergency. They must harness capabilities to augment their own by calling on the resources of others when required. The local authority will often have an emergency response centre which, in the event of an emergency, will provide information and support to the public (through emergency phone lines), to the responders and to the decision making authorities.

Across a range of emergency response scenarios, we can identify a common set for which intelligent agents might be of assistance:

- Sensor data management and fusion

- Accurate information gathering

- Correlation and validation

- Relevant and understandable communication

- Contact making

- $\quad$ Requests for assistance and matching to available capabilities

- Use of Standard Operating Procedures and Alarms

- $\quad$ Planning and coordination

- $\quad$ Scale and robustness

But one of the biggest challenges is to help these agents and services and the people using them to communicate and cooperate effectively. There are many instances in which lack of communication and breakdown in coordination has degraded the emergency response and in some cases led to further loss of life and property. 


\section{AI Challenges}

There are many AI challenges to be addressed to give such support and to make the vision a reality. Kitano and Tadokoro (2001) outlined some of this in a 50-year programme of work for future rescue robotics in Japan. They have also introduced the annual RoboCup Rescue Simulation competition held to test systems in a simulation of the 1995 Kobe earthquake. There have been several other proposals for "Grand Challenges" in computing and AI which take as their theme emergency response (Safety.net, 2002; I-Rescue Grand Challenges, 2006). As examples, the UK Advanced Knowledge Technologies (AKT) programme (Aktors, 2005) is addressing emergency response challenge problems, and a European follow-on programme called OpenKnowledge (2006) is using emergency response as one of its challenge problems. The FireGrid project is seeking to link sophisticated and large-scale sensor grids and faster than real-time simulations to emergency response coordination systems.

We can outline a number of core technologies, many having an essential AI component, which need to be developed, matured and integrated with other systems to make this vision of a connected world a reality. Examples of these technologies include:

1. Sensors and Information Gathering

a. sensor facilities, large-scale sensor grids

b. human and photographic intelligence gathering

c. information and knowledge validation and error reduction

d. Semantic Web and meta-knowledge

e. simulation and prediction

f. data interpretation

g. identification of "need"

2. Emergency Response Capabilities and Availability

a. robust multi-modal communications

b. matching needs, brokering and "trading" systems

c. agent technology for enactment, monitoring and control

3. Hierarchical, distributed, large scale systems

a. local versus centralized decision making and control

b. mobile and survivable systems

c. human and automated mixed-initiative decision making

d. trust, security

4. Common Operating Methods

a. shared information and knowledge bases

b. shared standards and interlingua

c. shared human-scale self-help web sites and collaboration aids

d. shared standard operating procedures at levels from local to international

e. standards for signs, warnings, etc.

5. Public Education

a. publicity materials 

b. self-help aids
c. training courses
d. simulations and exercises

Running through all these is the need for flexible and extendible representations of knowledge with rapidly altering scope, and with changing versions and refinements. There cannot be a single monolithically agreed representation of all the knowledge that will be involved. The science and technology of ontologies and their management will be vital to sustain this knowledge.

The technologies outlined above are drawn from a number of fields, some more mature than others, with each having its own philosophy and assumptions. However, the technological and research advances that are necessary to realize this vision are starting to be made in a number of projects and research programmes which will now be described.

\section{I-Rescue}

The I-Rescue project (I-Rescue, 2005) is exploring the use of AI planning and collaboration methods in rapidly developing emergency response and rescue situations. The overall aim is the creation and use of task-centric virtual organisations involving people, government and non-governmental organisations, automated systems, grid and web services working alongside intelligent robotic, vehicle, building and environmental systems to respond to very dynamic events on scales from local to global.

The I-X system and I-Plan planner (Tate et. al., 2004) provide a framework for representing, reasoning about, and using plans and processes in collaborative contexts. An underlying ontology, termed $<\mathrm{I}-\mathrm{N}-\mathrm{C}-\mathrm{A}>$ (Tate, 2003), is used as the basis of a flexible representation for the issues/questions to address, nodes/activities to be performed, constraints to be maintained and annotations to be kept. The I-X approach to plan representation and use relates activities to their underlying "goal structure" using rich (and enrichable) constraint descriptions which include the impact that the activities are meant to have on the state of the environment. This allows for more precise and useful monitoring of plan execution, allowing plans to be adjusted or repaired as circumstances change. It can make use of the dynamically changing context and status of the agents and products involved (e.g. through emerging geo-location services for people and products). It also provides for the real-time communication of activities and tasks between both human and automated resources.

I-X agents and the underpinning $<\mathrm{I}-\mathrm{N}-\mathrm{C}-\mathrm{A}>$ ontology can be used in a range of systems including supportive interfaces for humans and organisations, and potentially in intelligent sensors and robotic devices. It can thus act as a shared mechanism for coordinating these and for providing them with intelligent planning and process support. 
I-Rescue and I-X systems aim to be part of a future environment in which there are:

- Multi-level emergency response and aid systems

- Personal, vehicle, home, organisation, district, regional, national, international levels of assistance

- Backbone for progressively more comprehensive aid and emergency response

- Also used for aid-orientated commercial services

- Robust, secure, resilient, distributed system of systems

- Advanced knowledge and collaboration technologies

- Low cost, pervasive sensors, computing and communications

- Changes in building codes, regulations and practices.

\section{Coalition Agents Experiment (CoAX)}

As recent world events have shown, multi-national Coalitions play an increasingly important role in emergency response operations. The overall aim of is to exploit information better; in Coalitions this requires rapid integration of heterogeneous information handling and command systems, enabling them to inter-operate and share information coherently. However, Coalitions today suffer from labour-intensive information collection and co-ordination, and 'stove-piped' systems with incompatible representations of information.

The Coalition Agents Experiment (CoAX, 2006, Allsop et al., 2003) was an international collaborative research effort involving 30 organisations in four countries. It brought together a wide range of groups exploring agent technologies relevant to multi-national and multi-agency operations in the context of a peace-keeping scenario set in a fictional country - Binni (Rathmell, 1999). The principal research hypothesis was that the software agent technology and principles of the Semantic Web could help to initially construct and then use and maintain loosely coupled systems for complex and very dynamically changing Coalition operations (e.g. as in Wark et al., 2003). CoAX carried out a series of technology demonstrations based on a realistic Coalition scenario. These showed how agents and associated technologies facilitated run-time interoperability across the Coalition, adaptive and agile responses to unexpected events, and selective sharing of information between Coalition partners.

\section{Coalition Search and Rescue Task Support (CoSAR-TS)}

Search and rescue operations by nature require the kind of rapid dynamic composition of available policy-constrained services making it a good experimental basis for intelligent agent Semantic Web technologies. Semantic Web use within agents in CoAX was taken further in the CoSAR-TS project which also used the CoAX Binni scenario (Rathmell, 1999), and events which immediately followed on from those in the CoAX demonstrations. The KAoS agent domain management framework 
(Bradshaw et al., 1997) was used to describe the agent domains and the policies under which they interoperate. The project showcases intelligent agents and artificial intelligence planning systems working in a distributed fashion, with dynamic policies originating from various groups and individuals governing who is permitted or obligated to do what. The agents use Semantic Web services to dynamically discover medical information and to find local rescue resources. Semantic Web information access uses the OWL language (2004) and the rescue services are described in OWL-S (2005). I-X (Tate et al., 2002) was used as a task support, planning and execution framework to connect the various participants and services used. In later phases of the work, an exploration of web services composition using I-X's planner (I-Plan) was also included (Uszok et al., 2004).

\section{Collaborative Operations for Personnel Recovery (Co-OPR)}

Personnel recovery (search and rescue) teams must operate under intense pressure, taking into account not only hard logistics, but also "messy" factors such as the social or political implications of a decision. The "Collaborative Operations for Personnel Recovery” (Co-OPR) project has developed decision-support for sensemaking in such scenarios, seeking to exploit the complementary strengths of human and machine reasoning. Co-OPR integrates the Compendium (Buckingham-Sum et al., 2006) sensemaking-support tool for real time information and argument mapping, with the I$\mathrm{X}$ (Tate et al., 2002) artificial intelligence planning and execution framework to support group activity and collaboration. Both share a common model for dealing with issues, the refinement of options for the activities to be performed, handling constraints and recording other information. The tools span the spectrum with Compendium being very flexible with few constraints on terminology and content, to the knowledge-based reliance on rich domain models and formal conceptual models (ontologies) of I X. In a personnel recovery experimental simulation of a UN peacekeeping operation, with roles played by military planning staff, the Co-OPR tools were judged by external evaluators to have been very effective.

\section{Collaborative Advanced Knowledge Technologies e-Response}

The Collaborative Advanced Knowledge Technologies in the Grid (CoAKTinG) project (Buckingham Shum et al., 2002) used technologies from the UK Advanced Knowledge Technologies programme (Aktors, 2006) to support distributed scientific collaboration in an emergency response situation - in particular in a scenario involving the management of an oil spill. Focusing on the interchange between humans in the scenario, CoAKTinG provided tools to assist scientific collaboration by integrating intelligent meeting spaces, ontologically annotated media streams from on-line meetings, decision rationale and group memory capture, meeting facilitation, planning and coordination support, argumentation, and instant messaging/presence. 
The focus of AKT as a whole is on the provision of 'next generation' knowledge technologies, particularly in the context of the semantic web as both a medium and a target domain for these technologies.

New work on the AKT project is focused on a challenge problem dealing with the aftermath of a civil cargo aircraft crashing on a large city - an actual scenario for which there are existing contingency plans in place. It looks at how to use the semantic web to assist in making sense of the situation, both to guide emergency responders and to find appropriate specialized rescue and medical capabilities.

\section{OpenKnowledge e-Response}

OpenKnowledge (OpenKnowledge, 2006) is a European Union project involving Edinburgh, Southampton and the Open Universities in the UK, Amsterdam in The Netherlands, Trento in Italy and Barcelona in Spain. The goal of the project is to provide an open framework for agent interaction and coordination in knowledge-rich environments. It focuses on two challenge problems, one of which is emergency monitoring and management. This domain has been chosen as a testbed because it demands a combination of geographical and geo-presence knowledge alongside active support for collaboration and planning in multi-agent, dynamic situations.

The need to harness electronic communication networks in emergency situations has been recognised as a European research priority. Quoting from the EU "Emergency Response Grid” programme (EU, 2006):

"In times of crisis - be it a natural disaster, terrorist attack or infrastructure failure - mobile workers need to work together in time-critical and dangerous situations. Real-time access to information and knowledge, powered by Grids, will help save lives. Crises are complex situations, with large numbers and varieties of mobile workers - medical and rescue teams, police, fire fighters and other security personnel - appearing on the spot at short notice. These different teams come from different organisations, and generally have incomplete or even contradictory knowledge of the crisis situation.”

For OpenKnowledge the challenge of a test bed in this domain is in the rapidity of formation of emergency coalitions - often very intense and opportunistic "communities of practice" where judging the quality of an answer is critical and dynamic. OpenKnowledge involves the exploration of peer-to-peer services such as:

- Network data sources: Sensor data flows need to be coordinated in the large. These data will be classified locally and will need to be made available to different "experts/peers" on the basis of contextual classification schema.

- Collaborative services: to support planning, communication and coordination within the expert peers community. 
- Mitigation assessment services: as potential emergency situations are identified, mitigation needs can be determined and prioritized.

- Preparedness services: supporting those activities that prepare for actual emergencies.

\section{FireGrid}

A broad vision for an emergency response system in the modern built environment is being explored with an integrated and inter-disciplinary approach in FireGrid (Berry et al., 2005; FireGrid, 2006). This is a UK project to address emergency response in the built environment, where sensor grids in large-scale buildings are linked to faster-thanreal-time grid-based simulations, and used to assist fire responders to work with the building's internal response systems and occupants to form a team to deal with the emergency.

FireGrid will integrate several core technologies, extending them where necessary:

- High Performance Computing involving fire models and structural models

- Wireless sensors in extreme conditions with adaptive routing algorithms, including input validation and filtering

- Grid computing including sensor-guided computations, mining of data streams for key events and reactive priority-based scheduling

- Command and Control using knowledge-based planning techniques with user guidance

I-X will act as a "front-end" for the emergency response chief to access the various grid and web services which can be called upon to work with the human responders and those in the affected buildings.

\section{Helpful Systems and Helpful Organisations}

The infrastructure and organisations that would be required to make the vision of the helpful environment possible are being put in place. The Galileo European Satellite Navigation System (Galileo, 2006) and its mobile geo-location and emergency response services programme will be another spur to development. Both commercial and freely provided emergency response facilities are being interwoven to ensure active development and support over a long period.

Examples of the type of 'helpful organisation' that will enact this vision are already starting to emerge. An organisation called the Multinational Planning Augmentation Team (MPAT; Weide, 2006), consisting of 33 nations situated around the Pacific Rim, has been developing shared knowledge and procedures to assist in responses to regional crises. MPAT used computer collaboration aids, and a simple brokering 
system, during the December 2004 - February 2005 Indian Ocean Tsunami response to help affected countries gain access the specialized capabilities of response organisations more effectively. MPAT is an excellent example of people training and working together to ensure they are ready to respond better to emergencies. It would be a prime beneficiary and exploiter of any future 'helpful environment'.

On a smaller scale the Washington DC area is conducting a programme called Capital Wireless Integrated Network (CapWIN, 2005) led by IBM and the University of Maryland. This programme works with local services to support responses by all agencies to incidents occurring on a single freeway interchange in the DC area. Its aim is to show the potential value of coordinated wireless computing services for integrated and cohesive response across the police, the ambulance service, emergency support teams, the fire brigade, hazardous material units and the military.

\section{The Helpful Environment}

Imagine in the not too distant future that every citizen, vehicle, package in transit and other "active devices" can be treated as a potential sensor or responder. Individuals or vehicles that need help, as well as local, regional, national and international emergency agencies, could look up specialized capabilities or find local assistance through a much more responsive and effective environment. Systems could inter-operate to enable preventative measures to be taken so that those in imminent danger could be forewarned by their own systems, and by the people, vehicles and buildings around them.

These would support a diverse range of uses, such as:

- Disaster response and evacuation

- Terrorism incident response

- Civil accidents

- Disease control

- Business continuity

- Family emergencies

- Transportation aids

- Help desks

- Procedural assistance

A truly "helpful environment" could be created that is accessible by all.

Acknowledgments. This article is based on evidence given to US and UK government agencies concerned with responses to emergency situations and with "Grand Challenges" for computer science research. A version is included in the IEEE Intelligent Systems special issue on the Future of AI in 2006. Projects mentioned in this paper have been sponsored by a number of organisations. The University of Edinburgh and research sponsors are authorized to reproduce and distribute reprints 
and on-line copies for their purposes notwithstanding any copyright annotation hereon. The views and conclusions contained herein are those of the author and should not be interpreted as necessarily representing the official policies or endorsements, either expressed or implied, of other parties.

\section{References}

1. Aktors (2006) AKT: Advanced Knowledge Technologies.http://aktors.org

2. Allsopp, D.N., Beautement, P., Bradshaw, J.M., Durfee, E.H., Kirton, M., Knoblock, C.A., Suri, N., Tate A., and Thompson, C.W. (2002) Coalition Agents Experiment: Multi-Agent Co-operation in an International Coalition Setting, Special Issue on Knowledge Systems for Coalition Operations (KSCO), IEEE Intelligent Systems, Vol. 17 No. 3 pp. 26-35. May/June 2002.

3. Allsopp, D., Beautement, P., Kirton, M., Tate, A., Bradshaw, J.M., Suri, N. and Burstein, M. (2003) The Coalition Agents Experiment: Network-Enabled Coalition Operations, Special Issue on Network-enabled Capabilities, Journal of Defence Science, Vol. 8, No. 3, pp. 130-141, September 2003.

4. Berry, D., Usmani, A., Terero, J., Tate, A., McLaughlin, S., Potter, S., Trew, A., Baxter, R., Bull, M. and Atkinson, M. (2005) FireGrid: Integrated Emergency Response and Fire Safety Engineering for the Future Built Environment, UK e-Science Programme All Hands Meeting (AHM-2005), 19-22 September 2005, Nottingham, UK. http://www.aiai.ed.ac.uk/project/ix/documents/2005/2005-escience-ahm-firegrid.doc

5. Bradshaw, J M, Dutfield, S, Benoit, P, and Woolley, J D. (1997) KAoS: Toward an industrial-strength open agent architecture. In Software Agents, AAAI Press/MIT Press, Cambridge, Massachusetts, editor J M Bradshaw. Pages 375-418.

6. Buckingham Shum, S., De Roure, D., Eisenstadt, M., Shadbolt, N. and Tate, A. (2002) CoAKTinG: Collaborative Advanced Knowledge Technologies in the Grid, Proceedings of the Second Workshop on Advanced Collaborative Environments, Eleventh IEEE Int. Symposium on High Performance Distributed Computing (HPDC-11), July 24-26, 2002, Edinburgh, Scotland. http://www.aiai.ed.ac.uk/project/ix/documents/2002/2002-wacecoakting.pdf

7. Buckingham Shum, S., Selvin, A., Sierhuis, M., Conklin, J., Haley, C. and Nuseibeh, B. (2006). Hypermedia Support for Argumentation-Based Rationale: 15 Years on from gIBIS and QOC. In: Rationale Management in Software Engineering (Eds.) A.H. Dutoit, R. McCall, I. Mistrik, and B. Paech. Springer-Verlag: Berlin

8. CapWIN (2006) Capital Wireless Integrated Network. http://capwin.org

9. Co-OPR (2006) Coalition Operations for Personnel Recovery. http://www.aiai.ed.ac.uk/project/co-opr/

10. CoSAR-TS (2006) Coalition Search and Rescue Task Support. http://www.aiai.ed.ac.uk/project/cosar-ts/

11. CoAX (2006) Coalition Agents eXperiment http://www.aiai.ed.ac.uk/project/coax/

12. EU (2006) European Union Emergency Response Grid www.cordis.lu/ist/grids/emergencey_response_grid.htm

13. FireGrid (2006) FireGrid: The FireGrid Cluster for Next Generation Emergency Response Systems. http://firegrid.org

14. Galileo (2006) Galileo: European Satellite Navigation System. http://europa.eu.int/comm/dgs/energy_transport/galileo/

15. I-Rescue (2006) I-Rescue: I-X for Emergency Response and Related Grand Challenges. http://i-rescue.org 
16. Kitano, H. and Tadokoro, S. (2001) RoboCup Rescue: A Grand Challenge for Multiagent and Intelligent Systems, Artificial Intelligence Magazine, Spring, 2001, American Association of Artificial Intelligence.

17. MPAT (2006) Multinational Planning Augmentation Team Multinational Forces Standing Operating Procedures (MNF SOP) http://www2.apan-info.net/mpat/

18. OpenKnowledge (2006) OpenKnowledge.http://openk.org

19. OWL (2004) Ontology Web Language, World Wide Web Consortium. http://www.w3.org/2004/OWL/

20. OWL-S (2005) Ontology Web Language for Services. http://www.daml.org/services/owl-s/

21. Rathmell, R A. (1999) A Coalition force scenario 'Binni gateway to the Golden Bowl of Africa'. In Proceedings of the International Workshop on Knowledge-Based Planning for Coalition Forces, editor A Tate, pages 115-125, Edinburgh, Scotland, May 1999. Available at http://binni.org.

22. Safety Net (2002) Safety.net Grand Challenge Proposal. CRA Conference on "Grand Research Challenges" in Computer Science and Engineering Workshop, June 23-26, 2002, Warrenton, Virginia. http://www.cra.org/Activities/grand.challenges/slides/ubiquitous.pdf

23. Tate, A., Dalton, J., and J. Stader, J. (2002) I-P2- Intelligent Process Panels to Support Coalition Operations. In Proceedings of the Second International Conference on Knowledge Systems for Coalition Operations (KSCO-2002). Toulouse, France, April 2002.

24. Tate, A. (2003) <I-N-C-A >: an Ontology for Mixed-Initiative Synthesis Tasks. In Proceedings of the Workshop on Mixed-Initiative Intelligent Systems (MIIS) at the International Joint Conference on Artificial Intelligence (IJCAI-03), Acapulco, Mexico, August 2003.

25. Tate, A., Dalton, J., Siebra, C., Aitken, S., Bradshaw, J.M. and Uszok, A. (2004) Intelligent Agents for Coalition Search and Rescue Task Support, AAAI-2004 Intelligent Systems Demonstrator, in Proceedings of the Nineteenth National Conference of the American Association of Artificial Intelligence (AAAI-2004), San Jose, CA, USA, July 2004. http://www.aiai.ed.ac.uk/project/ix/documents/2004/2004-aaai-isd-tate-cosarts.pdf

26. Uszok, A., Bradshaw, J.M., Jeffers, R., Johnson, M., Tate, A., Dalton, J. and Aitken, S. (2004) KAoS Policy Management for Semantic Web Services, IEEE Intelligent Systems, pp. 32-41, July/August 2004.

27. Wark, S., Zschorn, A., Perugini, D., Tate, A., Beautement, P., Bradshaw, J.M. and Suri, N. (2003) Dynamic Agent Systems in the CoAX Binni 2002 Experiment, Special Session on Fusion by Distributed Cooperative Agents at the 6th International Conference on Information Fusion (Fusion 2003), Cairns, Australia, July, 2003.

28. Weide, S.A. (2006) Multinational Crisis Response in the Asia-Pacific Region: The Multinational Planning Augmentation Team Model, in "The Liaison", Center of Excellence in Disaster Management \& Humanitarian Assistance (COE-DMHA), February 2006. http://www.coe-dmha.org/liaison.htm 